ИЗВЕСТИЯ АКАДЕМИИ НАУК ЭСТОНСКОН ССР. ФИЗИКА * МАТЕМАТИКА

PROCEEDINGS OF THE ACADEMY OF SCIENCES OF THE ESTONIAN SSR.

PHYSICS * MATHEMATICS

$1986,35,4$

удК 539.199

P. KASK

\title{
SEPARATION OF NONORIENTATIONAL AND ROTATIONAL CONTRIBUTIONS IN FLUORESCENCE CORRELATION SPECTROSCOPY
}

\begin{abstract}
P. KASK. ORIENTATSIOONIST SOLTUMATU JA ROTATOORSE PANUSE ERALDAMINE FLUORESTSENTSKORRELATSIOONSPEKTROSKOOPIAS

П. КАСК. РАЗДЕЛЕНИЕ НЕОРИЕНТАЦИОННОГО И ВРАЩАТЕЛЬНОГО ФАКТОРОВ В ФЛУОРЕСЦЕНТНОЙ КОРРЕЛЯЦИОННОЙ СПЕКТРОСКОПИИ
\end{abstract}

(Presented by E. Lippmaa)

The first experiments in fluorescence correlation spectroscopy (FCS) $\left[{ }^{1}\right]$ induced a number of theoretical studies in which the fluorescence intensity autocorrelation function under continuous excitation was investigated. E. L. Elson and D. Magde $\left.{ }^{2}\right]$ considered translational and chemical processes responsible for fluorescence intensity fluctuations. In addition, M. Ehrenberg and R. Rigler $\left[{ }^{3,4}\right]$, and S. R. Aragon and R. Pecora $[5,6]$ considered the role of rotational diffusion in fluorescence intensity fluctuations. The expressions for the fluorescence intensity autocorrelation function also contain an anticorrelation term describing the dynamics of electronic transitions $\left[{ }^{3,4}\right]$. All these terms have been observed and used in experiments $\left[{ }^{1,7-9}\right]$.

As one can see, a number of existing different processes influence the fluorescence intensity autocorrelation function. It is of practical importance to separate the contribution of different processes in FCS experiments, particularly the contribution of the rotational motion from that of the other processes. This is the purpose of the present work. The theories about the separation of the rotational contribution, having been worked out in fluorescence dynamic depolarization (FDD) $\left[{ }^{10-12}\right]$ and fluorescence photobleaching recovery (FPR) $\left[{ }^{13-16}\right]$, serve as examples.

\section{Fluorescence intensity fluctuations in solution}

The fluorescence intensity from a microscopic sample at a given moment depends on the polarization of the exciting light (with unit electric vector $\hat{\mathbf{a}}$ ) and the polarization of the detection (with unit electric vector $\hat{\mathbf{e}}$, perpendicular to the direction of detection). For example, the probability density of the detection of photons emitted by a single fluorescent molecule fixed at a position $\mathbf{r}$ and at an orientation $\omega$, can be expressed as

$$
w(\mathbf{r}, \omega ; \hat{\mathbf{a}}, \hat{\mathbf{e}})=9 \sigma q \eta(\vartheta / 4 \pi) I(\mathbf{r}) U(\mathbf{r})\left[\hat{\mathbf{a}} \cdot \hat{\mu}_{a}(\omega)\right]^{2}\left[\hat{\mathbf{e}} \cdot \hat{\mu}_{e}(\omega)\right]^{2} .
$$

Here $\sigma$ is the isotropically averaged cross-section of absorption, $q$ is the quantum efficiency of fluorescence, $\eta$ is the quantum efficiency of detection, $\vartheta$ is the solid angle of detection, $I(\mathbf{r})$ is the exciting light intensity, $U(\mathbf{r})$ is the spatial distribution of the emitted light collection efficiency, 
$\hat{\mu}_{a}(\omega)$ is the unit vector of the absorption dipole, and $\hat{\mu}_{e}(\omega)$ is the unit vector of the emission dipole.

\section{Fluorescence intensity independent of label orientation}

There are several ways to eliminate the contribution of rotation motion in the fluorescence intensity fluctuations. The straightest way is to use spatially unpolarized excitation and detection. However, it is more difficult to realize than to imagine it. It is more convenient to use polarized excitation and detection. We denote the photon detection probability density at time $t$ and under given polarizations $\hat{\mathbf{a}}$ and $\hat{\mathbf{e}}$ as $w(t ; \hat{\mathbf{a}}, \hat{\mathbf{e}})$, and the correlation function

$$
\overline{w\left(t ; \hat{\mathbf{a}}^{\prime}, \hat{\mathbf{e}}^{\prime}\right) w(t+\tau ; \hat{\mathbf{a}}, \hat{\mathbf{e}})} \equiv G\left(\tau ; \hat{\mathbf{a}}^{\prime}, \hat{\mathbf{e}^{\prime}} ; \hat{\mathbf{a}}, \hat{\mathbf{e}}\right) .
$$

It is difficult to measure directly the correlation function given by Eq. (2), because it is not possible to measure simultaneously the fluorescence intensities corresponding to two different polarizations of excitation, $\hat{\mathbf{a}}^{\prime}$ and $\hat{\mathbf{a}}$. However, one can replace the continuous excitation by the pulse excitation at selected moments and selected polarizations, and the time average in Eq. (2) by the ensemble average. In the following it is assumed that the processes under study are much slower than the fluorescence lifetime, i.e. that the photon absorption and the photon emission occur simultaneously.

The fluorescence intensity independent of orientation, $w(t ; 0,0)$, can be obtained by taking the average from the intensities $w(t ; \hat{\mathbf{a}}, \hat{\mathbf{e}})$ over all possible unit vectors $\hat{\mathbf{a}}$ and $\hat{\mathbf{e}}$ :

$$
w(t ; 0,0)=(1 / 4 \pi)^{2} \iint w(t ; \hat{\mathbf{a}}, \hat{\mathbf{e}}) d^{2} \hat{\mathbf{a}} d^{2} \hat{\mathbf{e}} .
$$

(Every character «0» denotes an integration over the respective unit vector.)

Similarly, the autocorrelation function of the orientationally invariant fluorescence intensity can be expressed as

$$
G(\tau ; 0,0,0,0)=(1 / 4 \pi)^{4} \iiint \int G\left(\tau ; \hat{\mathbf{a}}^{\prime}, \hat{\mathbf{e}^{\prime}}, \hat{\mathbf{a}}, \hat{\mathbf{e}}\right) d^{2} \hat{\mathbf{a}}^{\prime} d^{2} \hat{\mathbf{e}}^{\prime} d^{2} \hat{\mathbf{a}} d^{2} \hat{\mathbf{e}}
$$

For practical goals, every integration in Eq. (4) (for example, over $\hat{\mathbf{a}})$ can be replaced by a summation over a small set, denoted by $\hat{\mathbf{a}}_{1}, \ldots, \hat{\mathbf{a}}_{n}$. This set must fulfil the condition

$$
\left(\hat{\mu} \cdot \hat{\mathbf{a}}_{1}\right)^{2}+\ldots+\left(\hat{\mu} \cdot \hat{\mathbf{a}}_{n}\right)^{2}=\operatorname{const}(\hat{\mu}) .
$$

This condition demands that the sum of the probabilities of excitation or detection should not depend on the direction of the transition dipole $\hat{\mu}$ of the molecule. One can choose a set of orthogonal unit vectors

or, alternatively,

$$
\hat{\mathbf{a}}_{1}=\hat{\mathbf{x}} ; \quad \hat{\mathbf{a}}_{2}=\hat{\mathbf{y}} ; \quad \hat{\mathbf{a}}_{3}=\hat{\mathbf{z}} ;
$$

$$
\begin{array}{ll}
\hat{\mathbf{a}}_{1}=3^{-1 / 2}(\hat{\mathbf{x}}+\hat{\mathbf{y}}+\hat{\mathbf{z}}) \equiv \hat{\mathbf{m}} ; & \hat{\mathbf{a}}_{2}=3^{-1 / 2}(\hat{\mathbf{x}}+\hat{\mathbf{y}}-\hat{\mathbf{z}}) \\
\hat{\mathbf{a}}_{3}=3^{-1 / 2}(\hat{\mathbf{x}}-\hat{\mathbf{y}}+\hat{\mathbf{z}}) ; & \hat{\mathbf{a}}_{4}=3^{-1 / 2}(-\hat{\mathbf{x}}+\hat{\mathbf{y}}+\hat{\mathbf{z}}) .
\end{array}
$$

It is experimentally possible to collect fluorescence light from the 
solid angle $2 \pi$ or $4 \pi$. In this case the correlation functions $G\left(\tau ; \hat{\mathbf{a}}^{\prime}, 0, \hat{\mathbf{a}}, 0\right)$ are directly measured and $G(\tau ; 0,0,0,0)$ can be expressed simply as

$$
G(\tau ; 0,0,0,0)=G(\tau ; \hat{\mathbf{z}}, 0, \hat{\mathrm{m}}, 0) .
$$

\section{Rotational contribution}

The treatment of rotational contribution in fluorescence intensity fluctuations is simplest if the ideal solution of a single, chemically stable fluorescent substance, is studied. In this case the correlation function can be expressed as a sum of the correlation functions for a single molecule, this is the time-dependent term, and of a time-independent term $\left[{ }^{17}\right]$ :

$$
\begin{aligned}
& G\left(\tau ; \hat{\mathbf{a}}^{\prime}, \hat{\mathbf{e}}^{\prime}, \hat{\mathbf{a}}, \hat{\mathbf{e}}\right)=\sum_{i} \sum_{j}\left\langle w_{i}\left(0 ; \hat{\mathbf{a}}^{\prime}, \hat{\mathbf{e}}^{\prime}\right) w_{j}(\tau ; \hat{\mathbf{a}}, \hat{\mathbf{e}})\right\rangle= \\
& =\sum_{i}\left\langle w_{i}\left(0 ; \hat{\mathbf{a}}^{\prime}, \hat{\mathbf{e}}^{\prime}\right) w_{i}(\tau ; \hat{\mathbf{a}}, \hat{\mathbf{e}})\right\rangle+\sum_{\substack{i \\
i \neq j}} \sum_{\substack{j\\
}}\left\langle w_{i}\left(0 ; \hat{\mathbf{a}}^{\prime}, \hat{\mathbf{e}}^{\prime}\right) w_{j}(\tau ; \hat{\mathbf{a}}, \hat{\mathbf{e}})\right\rangle= \\
& =G_{1}\left(\tau ; \hat{\mathbf{a}}^{\prime}, \hat{\mathbf{e}^{\prime}}, \hat{\mathbf{a}}, \hat{\mathbf{e}}\right)+\overline{w\left(\hat{\mathbf{a}}^{\prime}, \hat{\mathbf{e}}^{\prime}\right)} w(\hat{\mathbf{a}, \hat{\mathbf{e}})} \text {. }
\end{aligned}
$$

(The subscript index numbers the molecules.) Hence, only a single molecule has to be treated theoretically.

The time-dependent terms $G_{1}\left(\tau ; \hat{\mathbf{a}}^{\prime}, \hat{\mathbf{e}}^{\prime}, \hat{\mathbf{a}}, \hat{\mathbf{e}}\right)$ can be expressed through the conditional probability density of a molecule to be situated at the position $\mathbf{r}$ and at the orientation $\omega$ at time $t$, in case it was placed at the position $\mathbf{r}^{\prime}$ and at the orientation $\omega^{\prime}$ at zero time. The treatment of orientational motion is greatly simplified if it is assumed that this conditional probability density can be factorized as $R\left(\mathbf{r}, \mathbf{r}^{\prime}, \tau\right) \Omega\left(\omega, \omega^{\prime}, \tau\right)\left[{ }^{15}\right]$. Then the time-dependent term is expressed as

$$
\begin{aligned}
& G_{1}\left(\tau ; \hat{\mathbf{a}}^{\prime}, \hat{\mathbf{e}^{\prime}}, \hat{\mathbf{a}}, \hat{\mathbf{e}}\right)=C K \iiint \int \omega\left(\mathbf{r}^{\prime}, \omega^{\prime} ; \hat{\mathbf{a}}^{\prime}, \hat{\mathbf{e}}^{\prime}\right) \omega(\mathbf{r}, \omega ; \hat{\mathbf{a}}, \hat{\mathbf{e}}) R\left(\mathbf{r}, \mathbf{r}^{\prime}, \tau\right) \times
\end{aligned}
$$

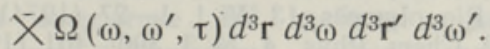

Here $C$ is the concentration of the label, and $K$ is a normalization constant.

The spatial and orientational variables can be separated and one obtains

$$
G_{1}\left(\tau ; \hat{\mathbf{a}}^{\prime}, \hat{\mathbf{e}}^{\prime}, \hat{\mathbf{a}}, \hat{\mathbf{e}}\right)=C K A \iint I(\mathbf{r}) I\left(\mathbf{r}^{\prime}\right) R\left(\mathbf{r}, \mathbf{r}^{\prime}, \tau\right) d^{3} \mathbf{r} d^{3} \mathbf{r}^{\prime} X
$$

$$
\begin{gathered}
\times \iint\left[\hat{\mathbf{a}}^{\prime} \cdot \hat{\mu}_{a}\left(\omega^{\prime}\right)\right]^{2}\left[\hat{\mathbf{e}}^{\prime} \cdot \hat{\mu}_{e}\left(\omega^{\prime}\right)\right]^{2}\left[\hat{\mathbf{a}} \cdot \hat{\mu}_{a}(\omega)\right]^{2}\left[\hat{\mathbf{e}} \cdot \hat{\mu}_{e}(\omega)\right]^{2} \Omega\left(\omega, \omega^{\prime}, \tau\right) d^{3} \omega d^{3} \omega^{\prime}= \\
=81 G_{1}(\tau ; 0,0,0,0) \times
\end{gathered}
$$$$
X\left\langle\left\{\hat{\mathbf{a}}^{\prime} \cdot \hat{\mu}_{a}[\omega(0)]\right\}^{2}\left\{\hat{\mathbf{e}}^{\prime} \cdot \hat{\mu}_{e}[\omega(0)]\right\}^{2}\left\{\hat{\mathbf{a}} \cdot \hat{\mu}_{a}[\omega(\tau)]\right\}^{2}\left\{\hat{\mathrm{e}} \cdot \hat{\mu}_{e}[\omega(\tau)]\right\}^{2}\right\rangle .
$$

$A$ is a constant of apparatus.

As it follows from Eq. (11), to separate the rotational factor in $G\left(\tau ; \hat{\mathbf{a}}^{\prime}, \hat{\mathbf{e}}^{\prime}, \hat{\mathbf{a}}, \hat{\mathbf{e}}\right)$, its time-dependent term has to be divided by the timedependent term of the orientationally invariant correlation function $G(\tau ; 0,0,0,0)$. 
The expression for the FPR signal ${ }^{[5]}$ can be written in the form similar to Eq. (11):

$$
\begin{gathered}
\Delta F(\tau ; \hat{\mathbf{p}}, \hat{\mathbf{a}}, \mathbf{e})=27 \Delta F(\tau ; 0,0,0) X \\
\left.X\left\{\hat{\mathbf{p}} \cdot \hat{\mu}_{p}[\omega(0)]\right\}^{2}\left\{\hat{\mathbf{a}} \cdot \hat{\mu}_{a}[\omega(\tau)]\right\}^{2} \hat{\mathbf{e}} \cdot \hat{\mu_{e}}[\omega(\tau)]\right\}^{2},
\end{gathered}
$$

where $\hat{p}$ is the unit vector of polarization of the bleaching pulse, and $\hat{\mu}_{p}$ the unit vector along the respective transition dipole. This expression is formally equivalent to the expression for the correlation function $G_{1}\left(\tau ; \hat{\mathbf{a}}^{\prime}, 0, \hat{\mathbf{a}}, \hat{\mathbf{e}}\right)$. One can also find the similarity between the expressions for the FDD signal and the correlation function $G_{1}(\tau: \hat{a}, 0,0, \hat{\mathbf{e}})$. In other words, the rotational factor of the FPR (or the FDD) signal is expressed as the average over one (or two) dimensions of a more detailed function, the rotational term of the FCS signal. Conseauently. of the three fluorescent methods to study orientational motion, the FCS is the most nowerful.

As it follows from Eq. (1), the amount of information about the orientation obtainable from $w(\mathbf{r}, \omega ; \hat{\mathbf{a}}, \hat{\mathbf{e}})$ is least meaningful if $\hat{\mu}_{a}=\hat{\boldsymbol{\mu}}_{\text {. }}$. Consequently, in FCS the fluorescent labels must be used for which $\hat{\mu}_{a} \neq \hat{\mu}_{e}$.

As we have seen, to sebarate the rotational contribution from the nonorientational one in FCS, the fluorescence intensity cross-correlation functions for different polarizations of excitation and detection have to be studied. The rotational factors of the cross-correlation functions carry along multifarious information about rotational motion and are therefore suggested to be taken into use in the rotational diffusion studies of unisotropic molecules or larger systems.

\section{R E F E R E N C E S}

1. Magde, D., Elson, E. L., Webb, W. W. Phys. Rev. Lett., 29, № 11, 705-708 (1972).

2. Elson, E. L., Magde, D. Biopolymers, 13. № 1. 1-27 (1974),

3. Ehrenberg, M., Rigler, R. Chem. Phys.. 4, № 3, 390-401 (1974).

4. Ehrenberg, M., Rigler, R. Quat. Rev. Biophvs., 9, № 1, 69-81 (1976).

5. Aragon, S. R., Pecora, R. Biopolymers. 14, № 1, 119-138 (1975).

6. Aragon, S. R., Pecora, R. J. Chem. Phys., 64, № 4, $1791-1803$ (1976)

7. Magde, D.. Elson, E. L.. Webb, W. W. Biopolvmers, 13, № 1, 29-61 (1974)

8. Kask, P., Piksarv, P., Mets, O. Eur. Biophys. J., 12, № 3, 163-166 (1985).

9. Kask. P., Piksarv, P., Mets. D., Pooga, M. Proc. Acad. Sci. ESSR. Phys. Math., 34, № $1, \quad 119-121 \quad(1986)$

10. Jablonski, A. Z. Physik, 95. 53-65 (1935).

11. Jablonski, A. Z. Naturforsch., 16A, $1-4$ (1961).

12. Tao, $T$. Bionolymers, 8, № 5, 609-632 (1969).

13. Smith, L. M., McConnell, H. M., Smith, B. A., Parce, J. W. Biophys, J., 33, № 1, $139-146$ (1981).

14. Johnson, P., Garland. P. B. FEBS Lett., 132, № 2, 252-256 (1981).

15. Wegener, W. A., Rigler, R. Biophys. J., 46, № 6, 787-793 (1984).

16. Wegener, W. A. Biophys. J., 46. № 6, 795-803 (1984).

17. Haas, E., Steinberg, I. Z. Biophys. J., 46, № 4, 429-437 (1984). 